Journal of the

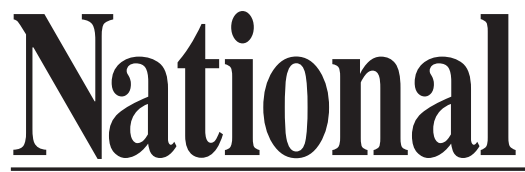

Academy or

Forensic
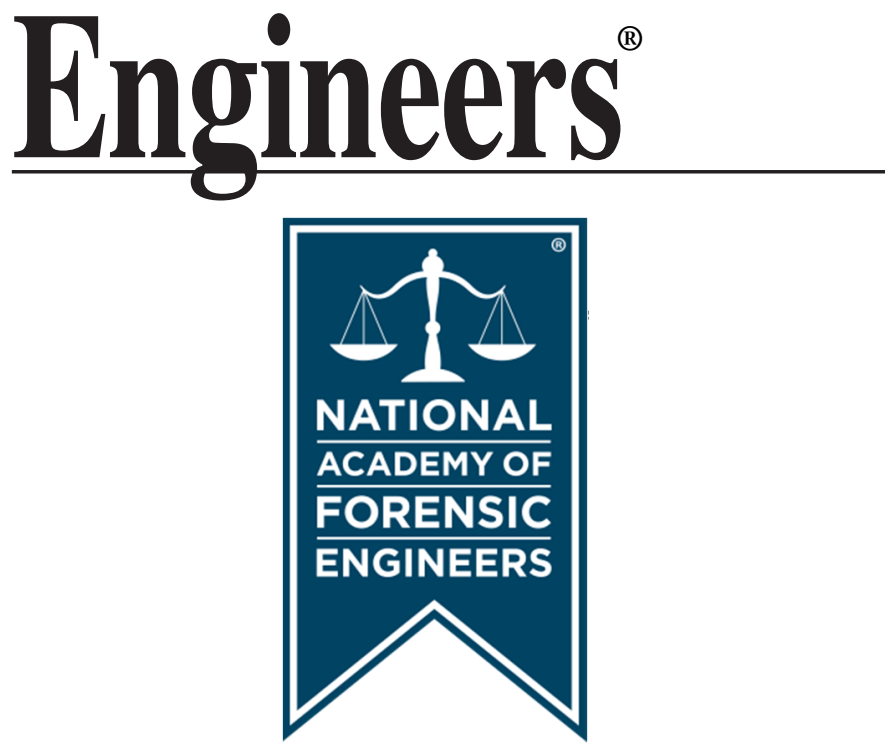

http://www.nafe.org ISSN: 2379-3252 


\title{
Forensic Engineering Investigation of Intentional Mis-Calibration of Vehicle Odometers
}

by Michael D. Leshner, P.E. (NAFE 559F)

\begin{abstract}
Odometers in motor vehicles record and display distance traveled. In addition to informing a driver of trip distance, this measurement is used to calculate service intervals, lease payments, resale value, fuel economy, and warranty limits. Distance is calculated from a digital accumulation of wheel rotations and knowledge of the tire circumference. The tire circumference may vary with speed, load, pressure, tread-wear and brand. This investigation compares indicated odometer measurements with simultaneous measurements using a Global Positioning System (GPS) device on a range of vehicle models. While some models have odometer systems with reasonably accurate calibrations, others systematically over-register odometer distance. In the opinion of this investigator, the mis-calibration is sometimes intentional and done for the economic benefit of the manufacturer.
\end{abstract}

\section{Background}

Odometer systems are measuring instruments and are generally assumed to be reasonably accurate. A range of factors affecting odometer accuracy will be discussed, along with strategies used by vehicle manufacturers to account and correct for sources of error.

Under nominal tire conditions, (correct size, pressure and nominal speed and load) modern electronic odometers are capable of excellent accuracy. However, in some cases, odometers are designed to run too fast, resulting in an economic loss to the consumer.

\section{Industry standards}

There are no Federal regulations in the U.S. regarding the accuracy of private vehicle odometers. Hawaii is the only State that addresses the issue, requiring +/- 3.75\% accuracy. The Society of Automotive Engineers (SAE) publishes a Recommended Practice, SAE J678 on the subject, and advises that the distance indication shall be $+/-4 \%$ of the actual distance traveled, at speeds of 20, 40 and $50 \mathrm{mph}$. This standard was issued in 1939 and reaffirmed in 1988. 
Copyright @ National Academy of Forensic Engineers (NAFE) http://www.nafe.org. Redistribution or resale is illegal. Originally published in the Journal of the NAFE volume indicated on the cover page. ISSN: 2379-3252

The National Institute of Standards and Technology (NIST) has published standards that apply to vehicles for rent or hire, requiring $+/-4 \%$ accuracy. The NIST standards warn against taking advantage of tolerances by calibrating to one side of zero, within the tolerance band.

\section{Mechanical odometers}

Before the advent of modern electronics in vehicles, odometers and speedometers were operated through a flexible, rotating mechanical cable linked to wheel rotation. By selecting appropriate gear ratios for the flexible cable take-off drive, a uniform relationship of 1,000 revolutionsper-mile was the industry standard. This relationship also provided a calibration point for mechanical speedometers where $1,000 \mathrm{rpm}$ produced an indication of $60 \mathrm{mph}$.

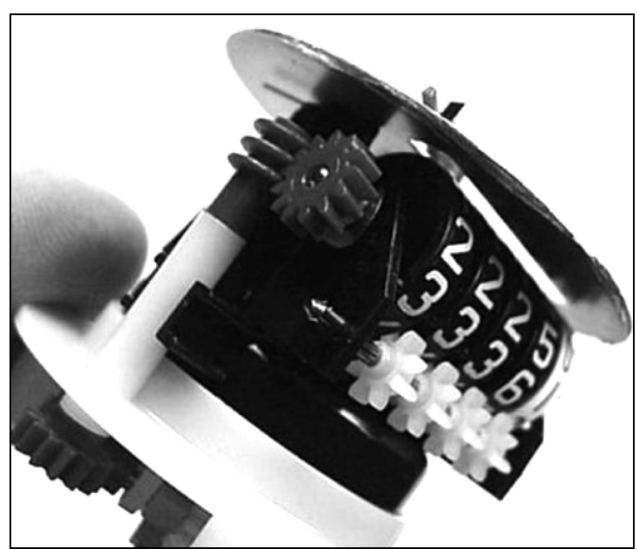

Figure 1

Typical mechanical odometer

Although mechanical speedometers were analog instruments subject to temperature changes, friction, slippage, drift and other sources of calibration errors, odometers have always been digital instruments. With direct gear-drive and no opportunity for slippage or drift, the accuracy of mechanical odometers depended only on adherence to the 1000 revolutions-per-mile standard. A typical mechanical odometer is shown in figure 1.

\section{Electronic odometers}

With the introduction of electronics into vehicles, odometer systems have evolved. Modern vehicles have an electronic, digital odometer display, with some form of pulse-generating sensor linked to one or more of the wheels. A typical wheel sensor is shown in figure 2 .

Most vehicles with anti-lock brake systems (ABS) make use of the ABS wheel sensors to generate the source of distance information. Other vehicles make use of a sensor elsewhere in the drive-line, generating pulses proportional to wheel rotations.

Regardless of sensor location, a fixed quantity of pulses are generated for each wheel revolution, and with knowledge of the dynamic tire circumference, a discrete number of pulses will be generated for each mile traveled. With a digi- 
tal signal at the sensing end of the odometer system, and a digital display in view of the driver, a digital calculator is interposed between the sensor and display. The calculator may be incorporated into the display or any of the multiple processors on the vehicle. Some vehicle systems also compensate for small changes in the dynamic tire circumfer-

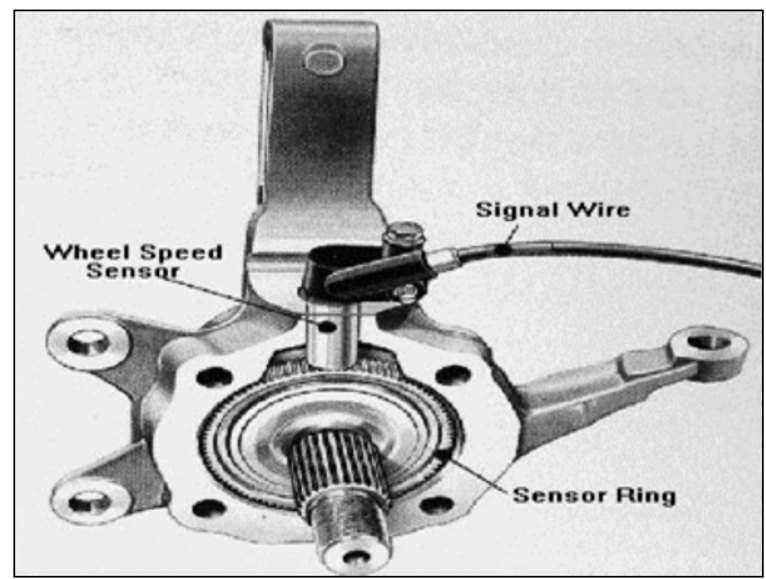

Figure 2

Typical wheel sensor ence as the speed changes, based on tire size measurements at varying speeds. To accurately indicate distance, the calculator must divide the source pulses by the correct number of pulses-per-mile.

\section{Factors affecting odometer accuracy}

Although the entire odometer system is digital, the dynamic tire circumference is subject to small variations with changing speed, age, brand, load, tread wear, and pressure. In order to evaluate odometer accuracy without the influence of these variables, testing can be performed under nominal conditions. The contribution of each of these variables can be measured separately by holding the others constant. Some examples will be discussed below.

Variations in tire circumference can be minimized by testing with new tires of the correct size, inflated to the correct pressure. Vehicle load and test speed can also be controlled. With nominal test conditions as a baseline, higher speeds cause the tires to grow, resulting in an odometer bias toward under-registration. Tread-wear, under-inflation and excess vehicle load all cause the tire circumference to become smaller, resulting in odometer over-registration.

\section{Test procedure}

Odometer accuracy may be evaluated by comparison to a reliable and accurate standard. The use of highway mile-markers has been found to be unreliable in tests of less than 20 miles. (Any individual marker can be in error by 50 feet or more.) Global Positioning System (GPS) devices have been found to be reliable and accurate. With resolution of 0.01 miles on a commercially available GPS device, an odometer test of 20 miles can be evaluated with uncertainty of less than one part in 20,000 , or 0.05 percent. 
By resetting the trip odometer and GPS to zero at the start of a test, both instruments accumulate distance simultaneously. The GPS accurately accumulates distance through turns and changes in direction, allowing both highway and city driving courses to be used. At the end of a test, the vehicle is allowed to continue rolling slowly, stopping when the tenth-mile digit on the odometer changes. Using this technique, potential odometer uncertainty of 0.1 mile is avoided.

Test data included model, year, VIN Number, tire size and brand, tread depth, tire pressure, date, time, type and location of test, odometer display and GPS measurement. A typical test result is shown in figure 3 .

\section{Test data}

The first set of tests involved six vehicles of one model suspected of odometer over-registration, spanning three model years. The vehicles were all tested on the same 20-mile highway course in both directions.

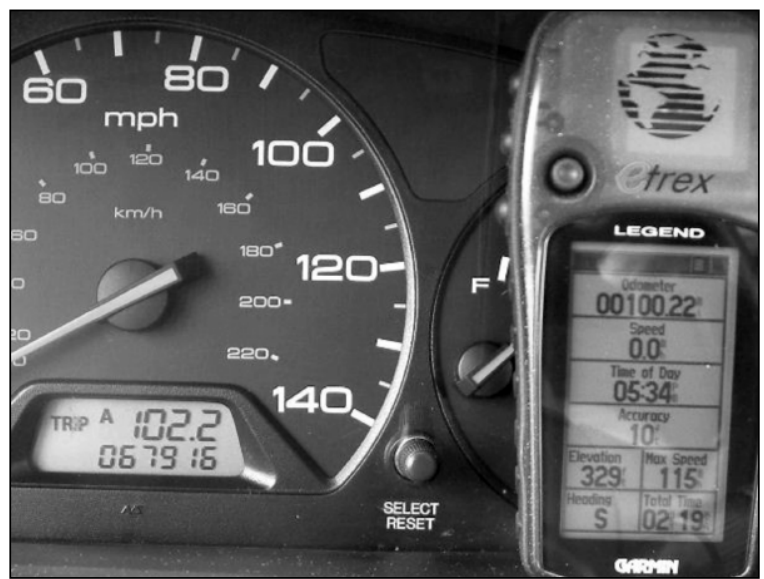

Figure 3

Typical test result

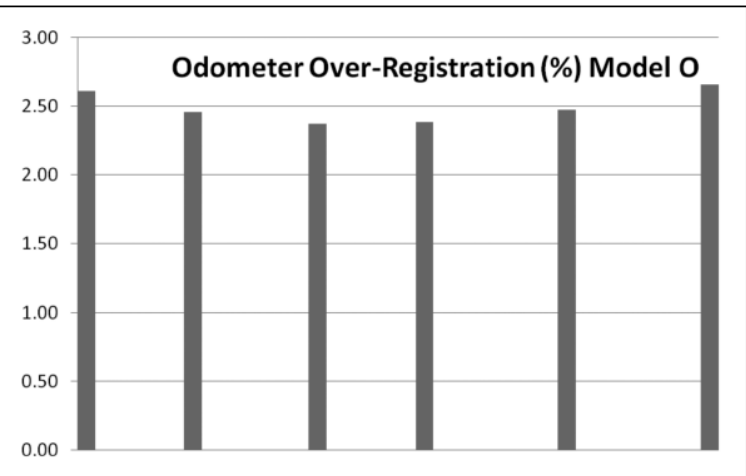

Figure 4

Six Vehicles - Model O

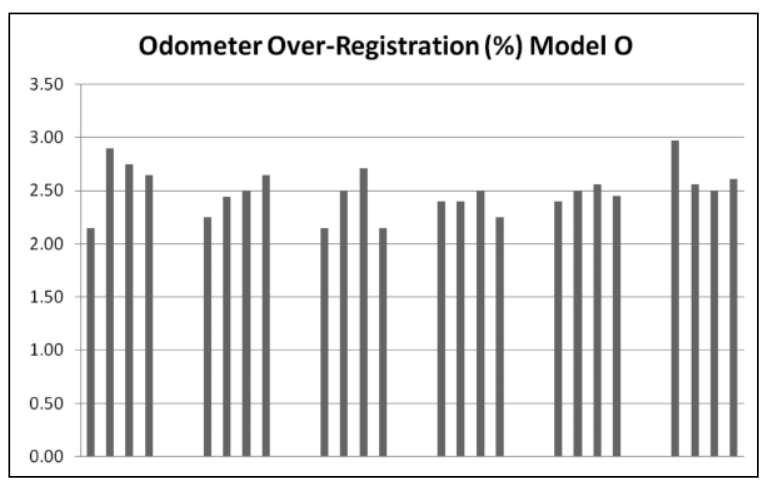

Figure 5

Six Vehicles, Four tests each 
Copyright @ National Academy of Forensic Engineers (NAFE) http://www.nafe.org. Redistribution or resale is illegal. Originally published in the Journal of the NAFE volume indicated on the cover page. ISSN: 2379-3252

Inspection of the data shows good uniformity of results among the six Model $\mathrm{O}$ test vehicles. Each bar on the chart in figure 4 represents the average

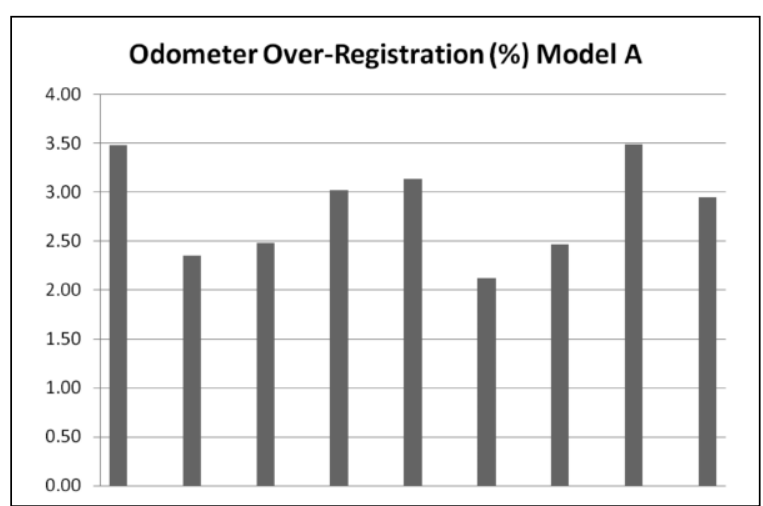

Figure 6

Nine Vehicles - Model A

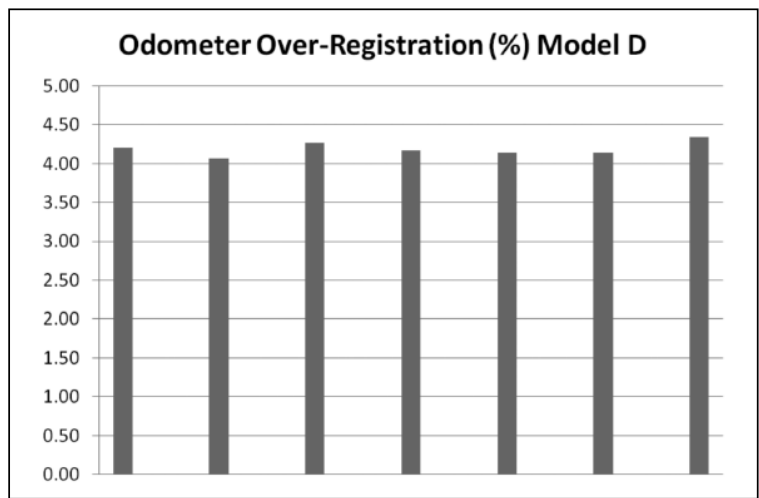

Figure 7

Seven Vehicles - Model D

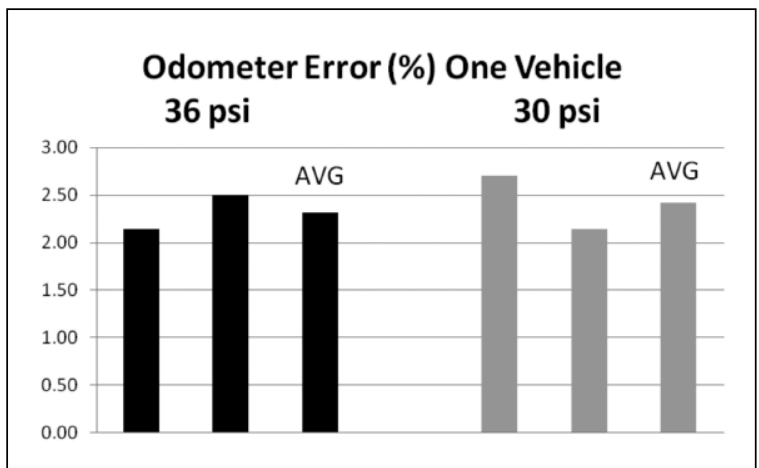

Figure 8

Effect of Tire Pressure of four tests.

The test-to-test repeatability was reasonably good, as shown in figure 5 .

The next model tested involved nine vehicles of the same model spanning three model years as shown in figure 6 . This model exhibited more variability between samples. The variability did not seem to be attributable to model year or tire brand.

The third model tested included seven vehicles spanning two model years. As shown in figure 7, vehicle-tovehicle uniformity was excellent on this model.

The influence of tire pressure was investigated, comparing the same vehicle with tires set to $30 \mathrm{psi}$ and 36 psi. As shown in figure 8 , the influence of a 6 psi difference in pressure was very small and less than test-to-test variability. 
The influence of test speed was also investigated. Highway tests at $70 \mathrm{mph}$ were compared with city driving at variable speeds in stop-and-go traffic. As shown in figure 9, odometer error was about one half percent greater at slower test speeds, due to the effect of speed on tire size.

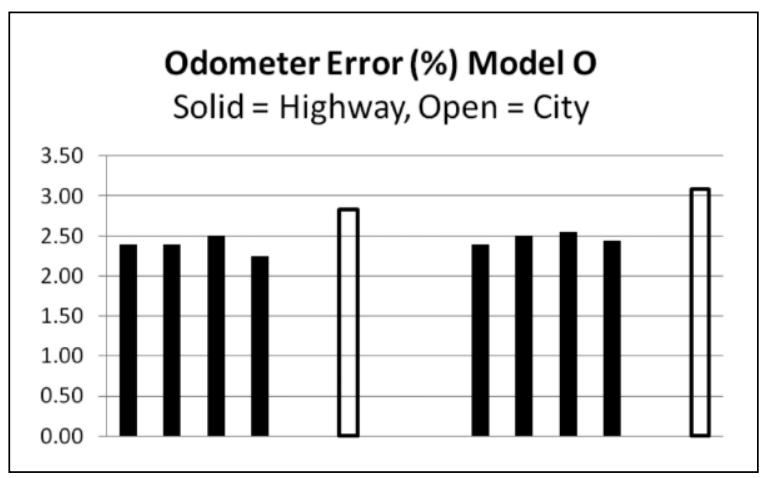

Figure 9

Effect of Driving Speed

\section{Class action lawsuits}

Class actions involving odometers have been initiated against at least five vehicle manufacturers, based on economic loss to customers. When odometers run fast, the loss to an individual owner is relatively small, but the gain to an auto manufacturer is substantial. The first lawsuit was against Honda and has settled, providing owners with extended warranties, refunded lease payments and some refunds for repairs that should have been covered under warranty.

One legal theory behind class action lawsuits for fast odometers is fraud. Proving that a manufacturer's vehicles have fast odometers is only the first step. The proof must also establish that the odometer system is capable of reasonable accuracy, if designed correctly. This kind of proof requires a thorough understanding of the odometer system design and implementation.

\section{Cracking the code}

Although the general theory underlying electronic odometers is straightforward, the actual implementation in a vehicle is often complex. Multiple factors need to be disclosed by the manufacturer and understood by the Forensic Engineer, including:

Source of distance data;

Dynamic tire circumference;

Arithmetic relationship between tire rotation and distance data;

Algorithms used to calculate distance;

Software used to communicate distance data, and

Manufacturer's internal standards for odometer accuracy. 
Copyright $\odot$ National Academy of Forensic Engineers (NAFE) http://www.nafe.org. Redistribution or resale is illegal.

Originally published in the Journal of the NAFE volume indicated on the cover page. ISSN: 2379-3252

NAFE 559F

MIS-CALIBRATION OF VEHICLE ODOMETERS

PAGE 83

With a thorough understanding of all these factors, the engineer can calculate the inherent system accuracy. For example, starting with 1,000 revolutions of the drive wheels, the actual and indicated distance can be calculated. Although one would expect those two results to be in good agreement, some manufacturers have "designed in" a positive bias of 2 to 4 percent, which is borne out in vehicle testing.

\section{Summary}

The rationale given by some manufacturers for odometer over-registration is that any result within + or -4 percent is within industry standards. However, the courts have disagreed with that position. Purposely calibrating odometers on the positive side of zero error has been determined to be fraud.

The Federal law against tampering with odometers (originally intended to apply to used cars) has also been interpreted by some courts to apply to vehicle manufacturers. The litigation is ongoing. 